\title{
Adam Neale (c1780-1832) and ergot of rye
}

\author{
Peter M Dunn
}

Adam Neale, MD, was born in Scotland around 1780. He was educated in Edinburgh and qualified in medicine in 1802 . In 1806 he was appointed as a physician to the army and saw considerable service during the Peninsula War under Sir John Moore and Sir Arthur Wellesley. In 1809 he published an account of his experiences in Letters from Portugal and Spain. In 1814 he settled in Exeter where he remained for the next 10 years apart from a tour of Germany, Poland, Moldavia, and Turkey in 1818 and a short sojourn in Cheltenham in 1820, made brief by the fact that he rashly and publicly questioned the medicinal value of the spa waters of that town. In 1828 he published a monograph on the use of ergot of rye as a remedy in certain states of the uterus. ${ }^{1}$ Four years later he died in Dunkirk in 1832.

Epidemics of St Anthony's fire due to ergot of rye had been recognised since the middle ages but the effect of ergot on the uterus was not recognised by the medical profession until 1688 when Camerarius mentioned that women in certain parts of Germany were in the habit of employing this sort of diseased grain to accelerate parturition. One hundred years passed before Tessier in France once more drew the attention of doctors to a similar practice among the midwives around Lyon. From then on medical interest in the subject increased and 50 years later Neale was able to review 720 reported cases in which ergot had been used in Europe and North America, mostly with success, either to expedite delivery of the baby or placenta or to control postpartum haemorrhage. Neale wrote:

'... the spurred rye or ergot which chiefly shows itself in wet seasons, and is generally considered to be a diseased change in the common grain . . . has been employed for a length of time in cases of lingering parturition ... when introduced into the stomach, or even the rectum, in doses of from 20 to 60 grains, it produces in a few minutes, by a stimulating or sympathetic action, strong and continued contractions of the uterus, followed by speedy and safe delivery ... provided always that the medicine be administered under suitable circumstances, and only when the labour has been delayed or suspended in consequence of feebleness of the womb'.

'The child being born, the womb continues to contract, closing upon itself, either by

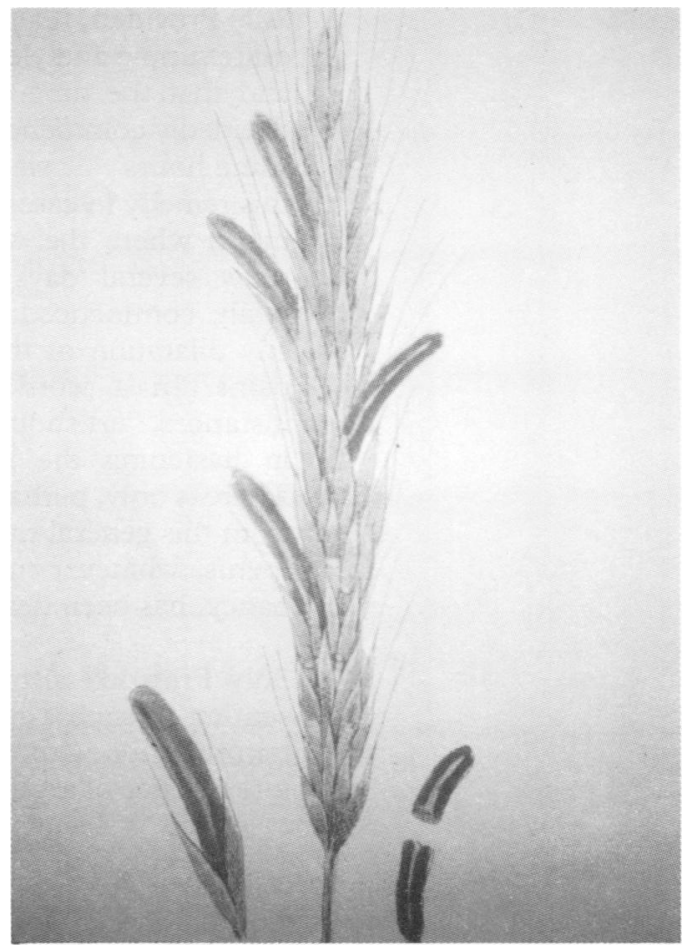

Ergot of rye, from the frontpiece of Adam Neale's book.

means of its natural contractability, or from the effect of the remedy ... we know of no case where the ergot has been employed a second time to procure the expulsion of the placenta, after having already been given to hasten the birth of the child ... we are induced to conclude that the uterine contractions produced by the ergot continue sufficiently long in all cases to bring about the expulsion of the placenta, as well as to hinder all subsequent hemorrhages'.

Neale was aware of the potential dangers of ergot to the mother and child when ergot was administered incorrectly or in inappropriate circumstances. As he wrote:

'But this substance has also been accused of injuring the child mechanically, and even of causing its death, in consequence of the violence of the uterine contractions thus artificially excited, which, it has been said, may even produce asphyxia, by obliterating the circulation between the placenta and the infant'.

Much of Neale's book is devoted to defining the situation in which ergot may be safely 
used and the contraindications to its administration:

'This remedy can only be administered with success in cases of parturition under particular circumstances, which may be classed under the following provisoes:

1st Provided always, that there exists no fault in the conformation of the bones of the pelvis, or of the soft parts of the mother, which can oppose any remarkable obstacle to the passage of the foetus. . .

2dly Provided, that the neck of the womb be quite supple and yielding, and partly dilated; and that the time of parturition shall have decidedly commenced, or already existed for some hours ... we ought not to administer this remedy in cases of premature accouchement; where the waters continue to drain away several days before parturition has really commenced; where there is neither any dilatation of the os uteri, nor any real pains. In a word, here, as in all other instances, "art should never precede nature" in hastening the natural act of delivery. There is only, perhaps, one case of exception from this general rule, and that is, where the foetus, whatever may be the state of pregnancy, has been dead for some time. . .

3dly Provided also, that the foetus is presenting in such a manner as to be expelled naturally; or without any necessity for the interference of art to change its position, and that its bulk is not too great for the natural passages . . . wherever parturition is considerably advanced, or the os uteri is sufficiently dilatable, and the lower extremities of the child are presenting, it would always be more advisable to pull down the feet, and deliver the woman than to try to excite the action of the womb by means of the spurred rye'.

Neale adds that ergot is contraindicated in a number of other circumstances including when: 'the umbilical cord be twisted several times round the neck of the foetus, and is thus causing an obstacle to delivery'. Maternal contraindications include convulsive and preconvulsive states. He writes:

'The most frequent obstacle which presents itself is a state of plethora, or turgidity of the blood vessels; as characterised by discoloration of the countenance, head-ache, fullness and hardness of the pulse, accompanied by strong uterine pains, without any expulsive efficacy. . . The actual presence, or even the threatening, of spasm and convulsion, either of the whole frame, or of the womb alone, form also a contraindication to the employment of spurred rye'.

Other contraindications included an extreme degree of nervous susceptibility, general debility, a liability to vomiting, and a past history of metritis or peritonitis.

1 Neale A. Researches respecting the natural history, chemical analysis and medicinal virtues of the spur, or ergot of rye, when administered as a remedy in certain states of the uterus. London, 1828. 